\title{
Linewidth, Autocorrelation, and Cross-Correlation Measurements of Counterpropagating Modes in GaAs-AlGaAs Semiconductor Ring Lasers
}

\author{
Guido Giuliani, Member, IEEE, Riccardo Miglierina, Student Member, IEEE, Marc Sorel, Member, IEEE, and \\ Alessandro Scirè
}

\begin{abstract}
We perform an experimental investigation of the linear autocorrelation and cross correlation properties of the two counterpropagating modes of a monolithic semiconductor ring laser (SRL). The ring laser can operate in a bidirectional regime where the two modes are active at the same time and have equal power, and also in a unidirectional regime where one of the modes is suppressed. Autocorrelation measurements are carried out using an unbalanced optical fiber Mach-Zehnder interferometer that allows us to determine the linewidth of each mode of the ring laser. Cross correlation measurements are performed using a modifed optical fiber interferometric setup where the two counterpropagating modes are directly superposed on a photodetector. This measurement reveals that in the bidirectional regime the two modes are phase-locked and allows us to evaluate the degree of their correlation.
\end{abstract}

Index Terms-Laser dynamics, laser linewidth, optical coherence, optoelectronic integrated circuits, ring laser, semiconductor laser.

\section{INTRODUCTION}

$\mathbf{S}$ EMICONDUCTOR ring lasers (SRLs) are attractive devices because they can be fabricated without the need for cleaved facet mirrors, offering the possibility of being monolithically integrated with other optoelectronic devices [1], [2]. SRLs can support two counterpropagating lasing modes that give rise to a rich phenomenology of operating regimes and dynamics. In particular, SRLs have been demonstrated to operate unidirectionally [3] with the possibility to select the active mode by means of electronic control [4], and also bidirectionally either in continuous wave $(\mathrm{CW})$ or in an interesting alternate oscillation regime [5], [6].

The scope of this work is to experimentally evaluate the coherence properties and the linewidth of the lasing modes in an SRL, as these have not been previously investigated in the literature. We have carried out experiments on SRL devices fabricated at the University of Glasgow in GaAs-AlGaAs material [6], designed to operate on a single transversal and longitudinal mode.

Manuscript received November 23, 2004; revised June 16, 2005.

G. Giuliani is with the Dipartimento di Elettronica, Università di Pavia, Pavia I-27100, Italy (e-mail: guido.guiliani@unipv.it).

R. Miglierina was with the Dipartimento di Electtronica, Università di Pavia, Pavia I-27100, Italy. He is now with ST Microelectronics, Cornaredo I-20010, Italy (e-mail: riccardo.miglierina@st.com).

M. Sorel is with the Department of Electronics and Electrical Engineering, University of Glasgow, Glasgow G12 8LT, U.K. (e-mail: sorel@elec.gla.ac.uk).

A. Scirè is with the Instituto Mediterraneo de Estudios Avanzados (IMEDEA), Universitat de les Illes Balears, Palma de Mallorca E-07122, Spain (e-mail: scire@imedea.uib.es).

Digital Object Identifier 10.1109/JSTQE.2005.854144

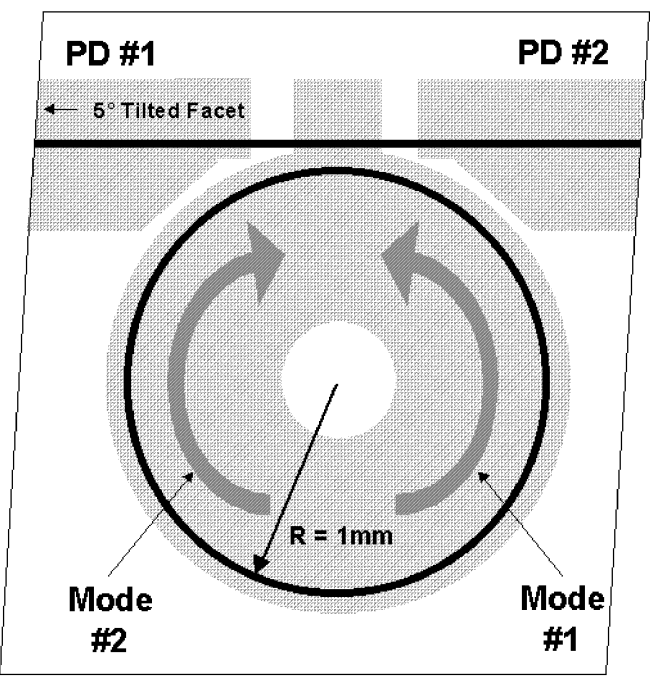

Fig. 1. Layout of the SRL waveguides and contacts.

The technique used to determine the linewidth of the SRL mode devices is based on fringe contrast measurement in an optical fiber Mach-Zehnder interferometer (MZI) when the unbalance of its two arms is varied [7].

The measured linewidth in the unidirectional regime is around $40 \mathrm{MHz}$, while in the bidirectional regime it is $110 \mathrm{MHz}$. Cross correlation measurements show that the two counterpropagating modes in the bidirectional regimes are phase-locked and the relative phase exhibits $\pi$ jumps [8].

\section{DEVICE DESCRIPTION}

The SRL devices are fabricated in GaAs-AlGaAs double quantum-well material emitting at $870 \mathrm{~nm}$ and have the structure shown in Fig. 1. The ring has a $1-\mathrm{mm}$ radius, and it is coupled via a directional coupler to a straight output waveguide that is tilted at $5^{\circ}$ to the cleaved end facets. Two separate contacts fabricated at the ends of the output waveguide are kept reverse biased, simultaneously serving two goals: 1) they act as integrated photodetectors, allowing the monitoring of the power of the two counterpropagating modes; and 2) they contribute to greatly reducing the amount of reflections generated at the output waveguide end facets. In fact, these reflections can be a major cause of coupling between the two counterpropagating modes, thus altering the mode dynamics that is intrinsic to the ring laser 


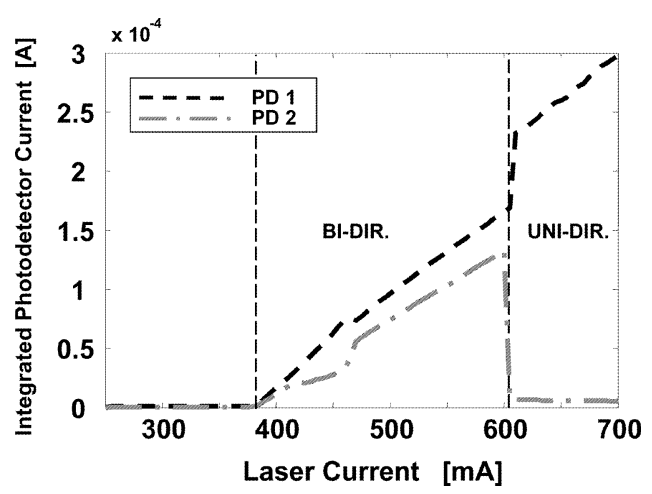

Fig. 2. P-I characteristic of the SRL device as measured through the integrated photodiode contacts. The device is kept at $25^{\circ} \mathrm{C}$, and the injected current is dc.

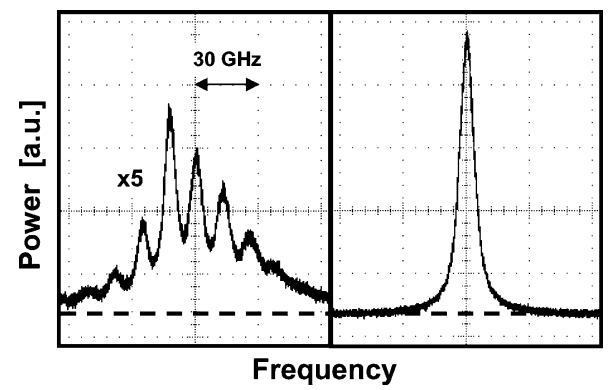

Fig. 3. Optical spectra of the SRL measured with Fabry-Perot filter (7 GHz FWHM). Left: ring current $=410 \mathrm{~mA}$, multiple longitudinal modes. Right: ring current $=500 \mathrm{~mA}$, single mode.

structure alone. We evaluate that the residual power coupling term due to end facets reflection is reduced to below $10^{-6}$.

Fig. 2 reports the experimental P-I characteristics of a typical SRL operated CW, showing a threshold value of $390 \mathrm{~mA}$. Just above threshold, a bidirectional regime is observed, where the two modes operate in $\mathrm{CW}$ and have nearby equal intensities. At higher currents, the regime becomes unidirectional, with one mode almost completely suppressed, while the other mode doubles its power. Typical attainable lasing mode power within the ring is around $5-50 \mathrm{~mW}$, and, as the power coupling ratio to the output waveguide can vary between $1 \%$ and $20 \%$ (depending on the spacing between the ring and straight waveguide), expected photocurrent levels measured at the integrated photodetectors are in the range of $0.025-5 \mathrm{~mA}$. All the fabricated SRLs exhibit wide operating regions where laser oscillation occurs on a single longitudinal mode, as demonstrated by the optical spectra acquired with a Fabry-Perot filter as shown in Fig. 3. Linewidth measurements have been carried out with the device operating on a single longitudinal mode.

\section{MeAsurement PRINCIPLE}

The technique chosen to determine the SRL linewidth is based on fringe visibility measurement in an unbalanced optical fiber MZI. Reasons that led to the choice of this measuring technique are: 1) the available modes' power outside the device is very small (due to the reverse-biased absorbing sections fabricated on the output waveguide ends, as discussed in Section II), hence preventing the use of most radio-frequency-based meth- ods such as the self-homodyne technique [9] or heterodyning; 2) the MZI technique measures the linear autocorrelation function of the mode field, conveying more information than the linewidth alone; and 3) a slight modification to the MZI setup also allows measurement of the cross correlation of the two counterpropagating modes in the bidirectional regime, which is of great interest in understanding SRL dynamics.

The experimental setup for the measurement of the autocorrelation and linewidth is shown in Fig. 4. The residual light not absorbed by the integrated photodetectors is emitted from both sides of the SRL, and it is butt-coupled to single-mode fibers, achieving launched power values around $1 \mu \mathrm{W}$. One fiber at a time is then connected to the main input of the MZI (labelled with the letter A in Fig. 4). The interferometer is made of two nominally 50-50 fiber couplers. Arm \#1 of the interferometer includes a polarization controller (PC) and an optical phase modulator fabricated by winding 10 fiber turns around a cylinder piezoceramic transducer (PZT). The PZT is driven by a $25-\mathrm{Hz}, 400-\mathrm{V}$ peak-to-peak triangle waveform so that the optical phase of the interferometer is modulated linearly with time. Arm \#2 has a variable length, which is varied by subsequently splicing together different spans of fiber. The optical pathlength difference of the interferometer arms is increasingly unbalanced, starting from 0 up to $10 \mathrm{~m}$. To obtain an accurate measurement of the optical pathlength difference of the interferometer arms, an external cavity laser (ECL) with a tunable emission wavelength is launched into the secondary input of the MZI (labelled with the letter B in Fig. 4). The wavelength of the ECL is swept linearly with time, and the frequency of the interferometeric signal detected by the photodiode allows retrieval of the effective unbalance of the MZI arms with an accuracy of a few centimeters. This calibration procedure is carried out each time the length of arm \#2 is varied.

The measurement principle is based on the fact that, when a laser mode is launched into the main input of the MZI and the optical phase is modulated by the PZT, a time domain interferometric signal is detected by the photodiode placed at the MZI output. The contrast (or fringe visibility) of this signal is a function of laser mode linewidth and of the MZI arms' unbalance. Repeated measurements for different unbalances allow retrieval of the linewidth.

The cross-correlation factor $\gamma_{c}(\tau)$ between two electromagnetic fields $E_{1}$ and $E_{2}$ is defined as

$$
\gamma_{c}(\tau)=\frac{\left\langle E_{1}(t) \cdot E_{2}^{*}(t-\tau)\right\rangle}{\left\langle\sqrt{\left|E_{1}(t) \cdot E_{1}^{*}(t)\right| \cdot\left|E_{2}(t) \cdot E_{2}^{*}(t)\right|}\right\rangle}
$$

where brackets denote the average over the optical cycle, and $\tau$ is the time equivalent to the pathlength difference between the MZI arms. When $E_{1}=E_{2}$, the above function is called autocorrelation, and it is denoted by $\gamma_{a}(\tau)$. For a laser mode with a Lorentzian lineshape, the autocorrelation is

$$
\gamma_{a}(\tau)=\exp \left(-\tau / \tau_{c}\right)
$$

where $\tau_{c}$ is the coherence time, from which the linewidth is calculated as $\Delta \nu=1 /\left(\pi \tau_{c}\right)$.

The interferometric signal (converted into a photocurrent) obtained at the output of the MZI is related to the mode 


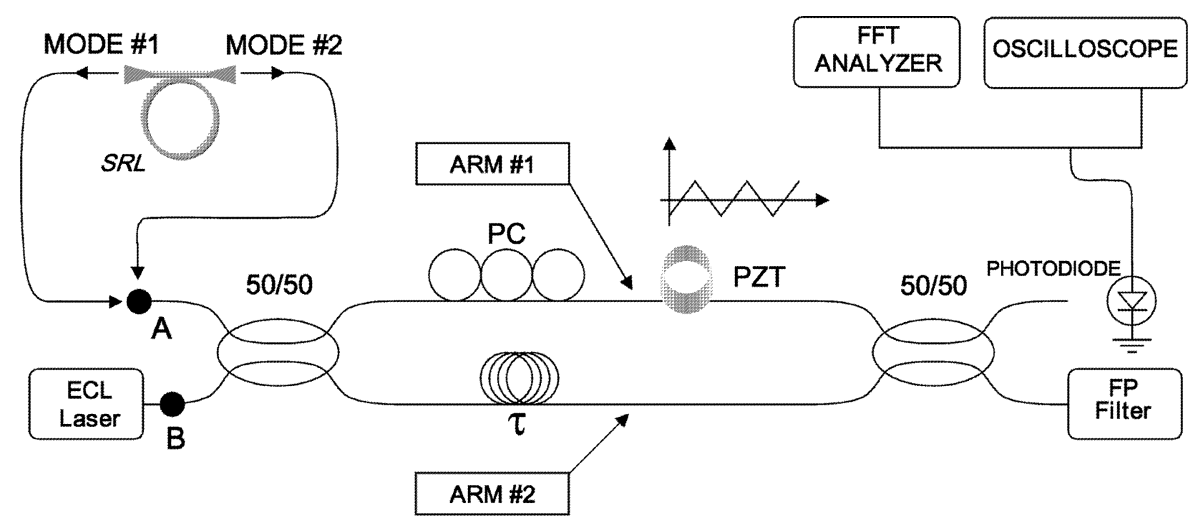

Fig. 4. Experimental setup for the measurement of the autocorrelation and linewidth of the SRL modes based on an optical fiber MZI. PC $=$ polarization controller; PZT = piezoceramic phase modulator driven by a triangle waveform; $\tau$ represents the propagation delay of arm \#2, which is varied by splicing different fiber spans.

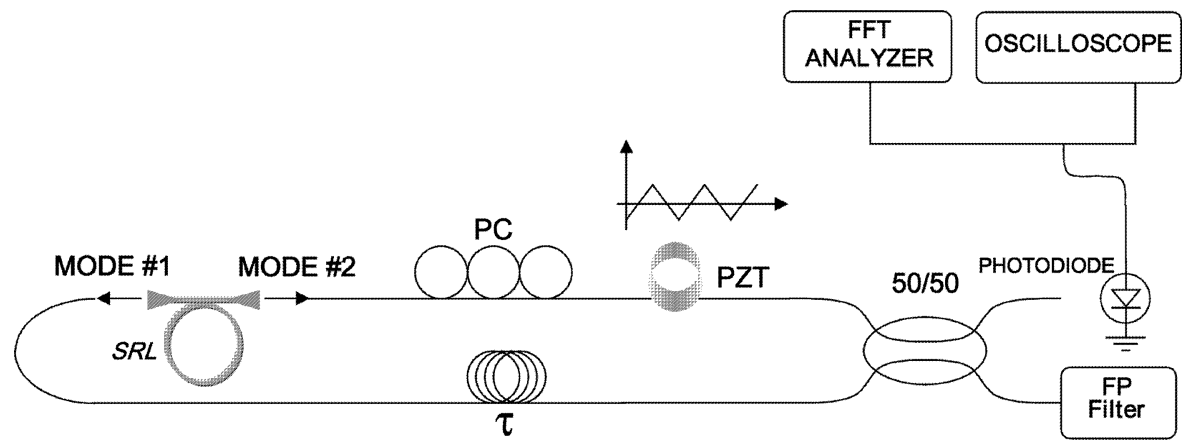

Fig. 5. Experimental setup for the measurement of the cross correlation between the two counterpropagating modes of the SRL in the bidirectional regime.

autocorrelation function by

$$
I_{\mathrm{ph}}=I_{0}\left[\frac{1}{2}+\frac{\left|\gamma_{a}(\tau)\right|}{2} \cdot \cos \phi(t)\right]
$$

where the interferometer phase $\phi(t)$ has the triangular waveform imposed by the PZT modulation, and the above result holds for the case of fiber couplers having exactly a 50-50 splitting ratio.

The contrast factor $C$ of the interferometric signal is defined as

$$
C=\frac{I_{\mathrm{ph}, \max }-I_{\mathrm{ph}, \min }}{I_{\mathrm{ph}, \max }+I_{\mathrm{ph}, \min }}
$$

yielding

$$
C=C(\tau)=\left|\gamma_{a}(\tau)\right|
$$

Hence, the contrast $C$ of the interferometric signal is a function of the MZI unbalance $\tau$, and it coincides with the autocorrelation function of the SRL mode. From a measurement of the contrast $C(\tau)$ for varying delay $\tau$, the autocorrelation function can be determined, from which the coherence time and linewidth can be obtained through a fitting procedure with the exponential function.

In practice, the actual value of the couplers splitting ratio deviates from 50-50, and the normalized contrast with respect to the case $\tau=0$ is used for the fitting procedure. Also, for MZI arm length in excess of a few meters, phase noise generated by thermal fluctuations of the fibers is superposed to the de- terministic interferometer phase $\phi(t)$, and the amplitude of the interferometric signal is more conveniently measured using an FFT spectrum analyzer instead of an oscilloscope.

The cross-correlation function $\gamma_{c}(\tau)$ between the two counterpropagationg modes of the SRL in the bidirectional regime can be measured by operating a slight modification to the experimental setup, obtaining the scheme shown in Fig. 5. The first fiber coupler of the MZI is removed, and the two lasing modes are simultaneously coupled into the two arms leading to the second coupler. In this manner, it is possible to determine the cross-correlation function $\gamma_{c}(\tau)$ by varying the arms' unbalance, once the fiber coupler splitting ratio has been characterized. If the two modes are uncorrelated, no interferometric signal is observed at the photodetector output, while the presence of the interferometric signal is evidence of a correlation.

\section{AUTOCORRELATION AND LINEWIDTH}

\section{A. Experimental Results}

Before proceeding to the measurement of the SRL linewidth, the MZI technique has been tested by characterizing the autocorrelation and linewidth of a single longitudinal mode FabryPerot semiconductor laser emitting at $825 \mathrm{~nm}$, obtaining results in good agreement with the $29-\mathrm{MHz}$ linewidth value measured with alternative methods [9]. The overall relative accuracy of the MZI method for linewidth determination is evaluated in $\pm 10 \%$. 
TABLE I

COHERENCE LENGTH $L_{c}$ AND Linewidth $\Delta \nu$ FOR THE Two COUNTERPROPAGATING MODES OF THE SRL As ObTAINED By EXPONENTIAL FitTing OF THE EXPERIMENTAL NORMALIZED CONTRAST CURVES REPORTED IN FIG. 6

\begin{tabular}{lll}
\hline & SRL Regime & \\
& Bidirectional & Unidirectional \\
Mode \#1 (counterclockwise) & $L_{c}=0.86 \mathrm{~m} ; \Delta V=111.0 \mathrm{MHz}$ & $L_{c}=2.52 \mathrm{~m} ; \Delta V=37.9 \mathrm{MHz}$ \\
Mode \#2 (clockwise) & $L_{c}=0.83 \mathrm{~m} ; \Delta V=115.0 \mathrm{MHz}$ & - \\
\hline
\end{tabular}

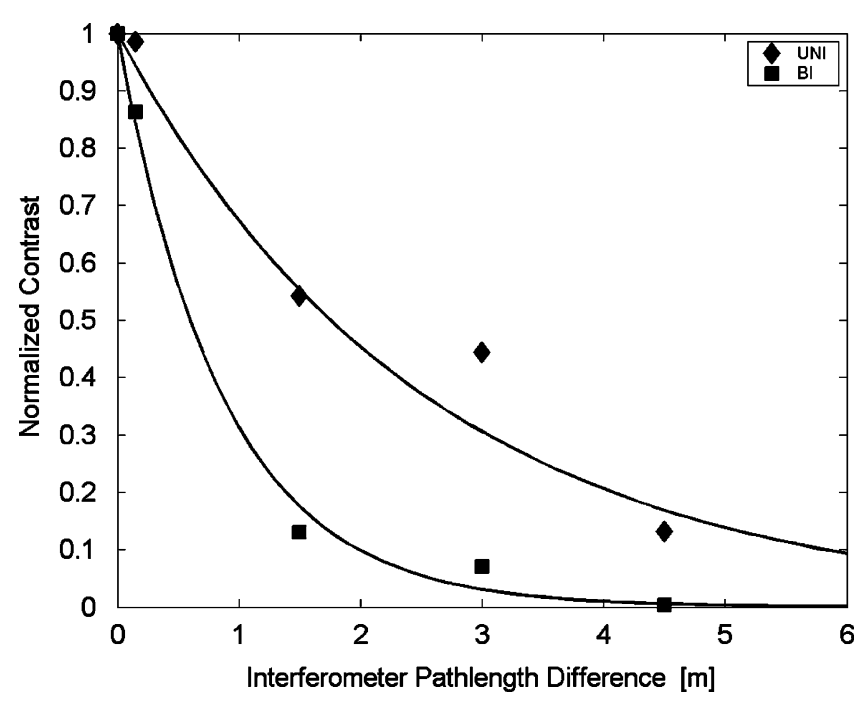

Fig. 6. Autocorrelation measurements for mode \#1 of the SRL. Symbols are measured data; lines are obtained by exponential fitting. Diamonds: unidirectional regime; squares: bidirectional regime.

Autocorrelation measurements are carried out when the SRL operates in bidirectional and unidirectional regimes, at current values very close to the transition between these two regions (i.e., around $600 \mathrm{~mA}$ ). In a unidirectional regime, the lasing mode is \#1 (counterclockwise).

Fig. 6 reports the measured values of the normalized contrast as a function of the interferometer pathlength difference $n \cdot c \cdot \tau$ (where $n$ is the fiber refractive index and $c$ is the speed of light) for mode \#1 when the laser is operated in the unidirectional regime, and for mode \#1 in the bidirectional regime (measurement for mode \#2 in this regime is identical). Lines are obtained by fitting the experimental data points with an exponential curve, from which coherence length and linewidth are derived. The values of coherence length and linewidth are reported in Table I.

\section{B. Theory}

We consider the Henry formula for the linewidth of a semiconductor laser [10], suitably modified for the case of a ring cavity laser that operates unidirectionally:

$$
\begin{aligned}
\Delta \nu & =\frac{v_{g}^{2} \cdot h \nu \cdot g_{\mathrm{mat}} \cdot \alpha_{m} \cdot n_{\mathrm{sp}, \mathrm{mat}} \cdot T \cdot\left(1+\alpha^{2}\right)}{4 \pi \cdot(T+L) \cdot P} \\
& =\frac{v_{g}^{2} \cdot h \nu \cdot\left(\alpha_{m}+\alpha_{w}\right) \cdot \alpha_{m} \cdot n_{\mathrm{sp}, \mathrm{mat}} \cdot T \cdot\left(1+\alpha^{2}\right)}{4 \pi \cdot(T+L) \cdot P}
\end{aligned}
$$

where $v_{g}$ is the group velocity; $g_{\text {mat, th }}$ is the material gain at threshold; $\alpha_{m}$ is the mirror loss; $P$ is the mode power coupled from the ring cavity to the output waveguide; $\alpha$ is the linewidth enhancement factor; and $L$ and $T$ are, respectively, the excess loss of the output coupler and its power transmission to the output waveguide. The material spontaneous emission factor is defined as $n_{\text {sp,mat }}=\gamma_{\text {mat }} /\left(\gamma_{\text {mat }}-\alpha_{\text {mat }}\right)$ [10], where $\gamma_{\text {mat }}$ and $\alpha_{\text {mat }}\left[\mathrm{cm}^{-1}\right]$ are, respectively, the net stimulated emission and net absorption coefficients of the active material. At threshold, the material gain equals $\left(\alpha_{m}+\alpha_{w}\right)$, where $\alpha_{w}$ is the waveguide loss. For an SRL, the mirror loss is defined as

$$
\alpha_{m}=-\frac{\ln (1-T-L)}{2 \pi R}
$$

where $R$ is the ring radius. For our large-radius SRLs, the mirror loss is around $2-3 \mathrm{~cm}^{-1}$, and hence it is much smaller than the waveguide loss, in opposition to most linear edge-emitting semiconductor lasers. The material spontaneous emission factor is in the range of 2.5-3.5 for lasers that exhibit relatively high total cavity losses, and consequently require a high threshold gain. This is the case of buried waveguide Fabry-Pérot lasers, for which total cavity losses (mirror loss + waveguide loss) can be about $60-90 \mathrm{~cm}^{-1}$, and of SRLs with high waveguide loss. Conversely, SRLs with low waveguide loss require a low inversion of the active medium to reach the threshold condition, and $n_{\text {sp }, \text { mat }}=8-10$.

For the SRL considered in the present work, reasonable values for the above parameters are $\alpha_{w}=80 \mathrm{~cm}^{-1}, n_{\mathrm{sp} \text {, mat }}=$ $3.5, T=0.3, L=0.5, \alpha=5.5$, and $P=0.3 \mathrm{~mW}$. A numerical evaluation of the linewidth of the SRL according to (6) yields $38.5 \mathrm{MHz}$, in agreement with the measured value in the unidirectional regime. The above parameters also allow us to evaluate the SRL differential quantum efficiency as

$$
\eta_{q}=\frac{\alpha_{m}}{\alpha_{m}+\alpha_{w}} \cdot \frac{T}{T+L}=0.0116
$$

in agreement with the experimental value $\eta_{q}=0.010$. The SRL considered here has a ridge depth beyond the optimum value [6], which causes excess waveguide loss, and consequently a large linewidth. Other SRLs fabricated from the same wafer with an optimum ridge depth show a lower threshold current [6] and reduced waveguide loss, and a linewidth close to $1 \mathrm{MHz}$ can be obtained (i.e., for $\alpha_{w}=15 \mathrm{~cm}^{-1}, n_{\mathrm{sp} \text {, mat }}=8, P=5 \mathrm{~mW}$ ).

A comparison of the measured linewidth in the two operating regimes is worthwhile. The linewidth in the unidirectional regime is smaller than in the bidirectional regime, but a closer 
look to the quantitative aspect reveals some interesting features. For mode \#1, we can define the product mode intensity times linewidth as $F_{1}=P_{1} \cdot \Delta \nu_{1}$. According to (7), we would expect this product to be constant, as no important change in threshold gain is expected between the two regimes. However, when we calculate this product for the two regimes of the SRL, we find $F_{1, \mathrm{UNI}}=P_{1, \mathrm{UNI}} \cdot \Delta \nu_{1, \mathrm{UNI}}=13.1 \mathrm{~mW} \cdot \mathrm{MHz}$, and $F_{1, \mathrm{BI}}=P_{1, \mathrm{BI}} \cdot \Delta \nu_{1, \mathrm{BI}}=25.2 \mathrm{~mW} \cdot \mathrm{MHz}$. Hence, in the bidirectional regime, the product mode intensity times linewidth is almost doubled with respect to the unidirectional regime. From (6), it seems hardly possible that the threshold gain $g_{\text {th }}$ or the spontaneous emission factor $n_{\mathrm{sp}}$ can vary by such a large amount between the two regimes. So, it can be argued that when the two counterpropagating modes are simultaneously active within the ring cavity, each mode produces a sensible increase in the linewidth of the other mode (with respect to the case of a solitary mode with the same power) through the phase-amplitude coupling mechanism represented by the linewidth enhancement factor $\alpha$. This means that in a bidirectional SRL the amplitude noise of one mode influences the phase fluctuations of the other mode.

\section{CRoss Correlation}

First, using the experimental setup of Fig. 5, the cross correlation between the two counterpropagating modes of the SRL is investigated in the bidirectional regime for $\tau=0$ (i.e., balanced pathlengths). For the case of two uncorrelated modes, the output would be only noise, with no trace of interferometric signal. In our experiment, a stable interferometric sinusoidal signal is observed on the photodiode when the PZT is modulated by the triangle waveform. This is clear evidence that the two counterpropagating modes are frequency-locked and phaselocked in the bidirectional regime. After some time (typically 5-10 s), a sudden jump in the value of the relative phase may occur, and the modes get locked with a new value of the relative phase that differs from the previous one by $\pi$. This is proved in Fig. 7, which reports the time trace of the interferometric signal acquired by a digital oscilloscope with a long persistence $(10 \mathrm{~s})$. The trigger instant (i.e., the instant at which the triangle waveform applied to the PZT changes slope) is located at the center of the screen. During this acquisition, a phase jump occurs, and the picture shows two distinct time traces corresponding to different values of the lock-in phase, with the phase difference $\approx \pi$. An explanation for the $\pi$ jumps in the lock-in phase between the two modes can be made on the basis of the analysis of injection-locked laser systems [11]. The SRL has the peculiarity that the two phase-locked travelling counterpropagating modes give rise to a standing wave pattern that has in principle no spatial boundary conditions that impose a specific phase value at some point. As a consequence, two degenerate standing wave patterns that are offset by $\lambda / 4$ are equally probable, and the $\pi$ jumps in the relative phase of the two travelling modes [8] correspond to different standing wave patterns.

Complete cross-correlation measurements are then performed by varying the length of arm \#2. The result of the measurement

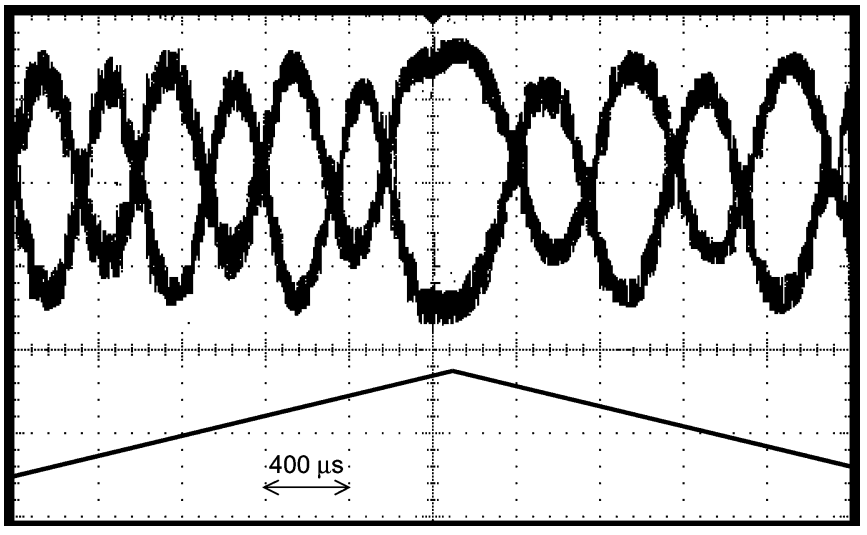

Fig. 7. Upper trace: interferometric signal obtained with the setup of Fig. 5 in bidirectional regime. Lower trace: PZT drive waveform. Time scale: $400 \mu \mathrm{s} / \mathrm{div}$ The oscilloscope persistence is $10 \mathrm{~s}$. The upper trace is the superposition of two different situations, corresponding to a $\pi$ jump of the lock-in phase between the two counterpropagating modes, that occurred during the acquisition.

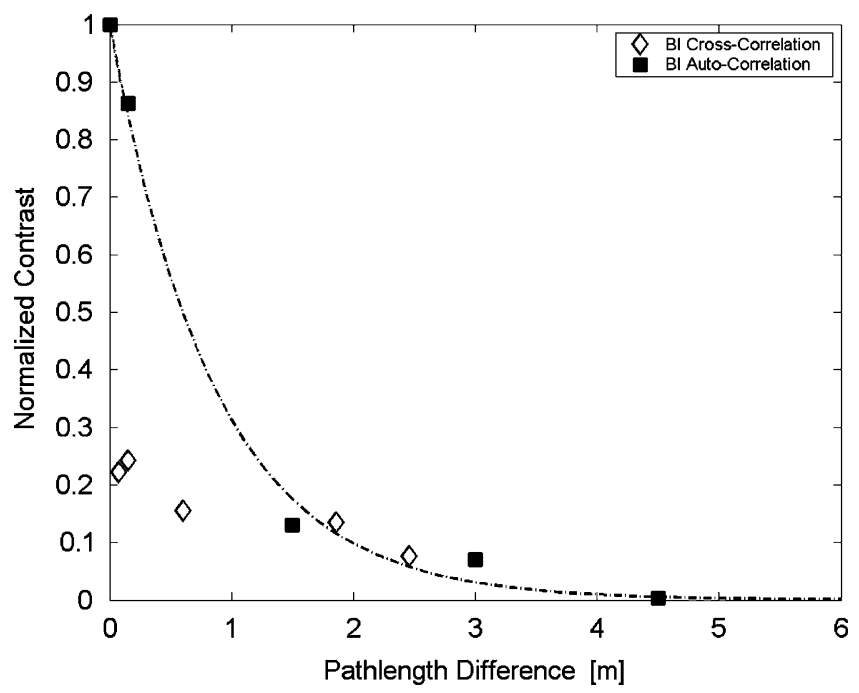

Fig. 8. Cross-correlation measurement of mode \#1 and mode \#2 of the SRL in the bidirectional regime (open diamonds). For comparison, the autocorrelation data measured in the bidirectional regime for mode \#1 are also reported (squares: data; dash-dotted line: exponential fitting).

is reported in Fig. 8. In this picture, cross-correlation data (diamonds) are plotted along with autocorrelation data previously measured for the bidirectional regime. As expected, the cross correlation between mode \#1 and mode \#2 is always smaller than the autocorrelation of either mode \#1 or mode \#2. From the data in Fig. 8, it is not straightforward to determine the degree of correlation of the two fields. In fact, if we define the degree of correlation as the ratio of the cross-correlation to the autocorrelation, this parameter shows a dependence on the delay $\tau$. For $\tau=0$, the degree of correlation is around 0.23 , while for larger values of $\tau$ it approaches unity. Interpretation of these data requires further studies about the field statistics of the two modes. 


\section{CONCLUSION}

We have reported experimental investigations of the linewidth and coherence properties of monolithic SRLs. The linewidth of the SRL modes has been measured through an unbalanced fiber MZI under different operating regimes: unidirectional and bidirectional. Measured linewidth value has been successfully related to the theoretical expression. Cross correlation measurements between the two counterpropagating modes in the bidirectional regime have revealed that the modes are phase-locked and are correlated to a high degree.

\section{ACKNOWLEDGMENT}

The authors would like to thank Prof. S. Donati for stimulating discussions about this work.

\section{REFERENCES}

[1] T. F. Krauss, P. J. R. Laybourn, and J. S. Roberts, "CW operation of semiconductor ring lasers," Electron. Lett., vol. 26, no. 25, pp. 20952097, Dec. 1990.

[2] T. F. Krauss, R. M. De La Rue, P. J. R. Laybourn, B. Vogele, and C. R. Stanley, "Efficient semiconductor ring lasers made by a simple self-aligned fabrication process," IEEE J. Sel. Topics Quantum Electron., vol. 1, no. 2, pp. 757-761, Jun. 1995.

[3] J. P. Hoimer and G. A. Vawter, "Unidirectional semiconductor ring laser with racetrack cavities," Appl. Phys. Lett., vol. 63, pp. 2457-2459, Nov. 1993.

[4] M. Sorel, G. Giuliani, S. Donati, and P. J. R. Laybourn, "Unidirectional bistability in semiconductor waveguide ring lasers," Appl. Phys. Lett., vol. 80, pp. 3051-3053, Apr. 2002.

[5] M. Sorel, P. J. R. Laybourn, A. Scirè, S. Balle, G. Giuliani, R. Miglierina, and S. Donati, "Alternate oscillations in semiconductor ring lasers," Opt. Lett., vol. 27, no. 22, pp. 1992-1994, Nov. 2002.

[6] M. Sorel, G. Giuliani, A. Scirè, R. Miglierina, S. Donati, and P. J. R. Laybourn, "Operating regimes of GaAs-AlGaAs semiconductor ring lasers: Experiment and model," IEEE J. Quantum Electron., vol. 39, no. 10, pp. 1187-1195, Oct. 2003.

[7] D. Derickson, Ed., Fiber Optic Test and Measurement. Englewood Cliffs, NJ: Prentice-Hall, 1997.

[8] T. H. Chyba, "Phase-jump instability in the bidirectional ring laser with backscattering," Phys. Rev. A, Gen. Phys., vol. 40, pp. 6327-6338, Dec. 1989.

[9] T. Okoshi, K. Kikuchi, and A. Nakayama, "Novel method for high resolution measurement of laser output spectrum," Electron. Lett., vol. 16, no. 16 , pp. 630-631, Jul. 1980.

[10] C. H. Henry, "Theory of the linewidth of semiconductor lasers," IEEE J. Quantum Electron., vol. 18, no. 2, pp. 259-264, Feb. 1982.

[11] V. Annovazzi-Lodi, S. Donati, A. Scirè, and M. Sorel, "Dynamic behavior and locking of a semiconductor laser subjected to external injection," IEEE J. Quantum Electron., vol. 34, no. 12, pp. 2350-2357, Dec. 1998.
Guido Giuliani (M'99) was born in Milan, Italy, in 1969. He received the Ph.D. degree in electronics and computer science from the Università di Pavia, Pavia, Italy, in 1997.

Since 2000, he has been an Assistant Professor in Optoelectronics at the Università di Pavia. His research interests include semiconductor lasers, semiconductor optical amplifiers, optical amplifier noise, and interferometry and optoelectronic sensors.

Riccardo Miglierina (SM'99) received the Laurea degree in electronic engineering in 2001 and the Ph.D. degree in electronics and computer science in 2004 from the Università di Pavia, Pavia, Italy.

His research interests include characterization and modeling of semiconductor lasers and high bandwidth photodetectors. Since 2005, he has been with ST Microelectronics, Milan, Italy.

Marc Sorel (M'99) received the Laurea degree in electronics engineering and the Ph.D. degree in electronics and computer science from the Università di Pavia, Pavia, Italy, in 1995 and 1999, respectively.

In 1998, he joined the Optoelectronics Group at the University of Glasgow, Glasgow, U.K., and he was recipient of a Marie Curie Fellowship in 1999 for research on integrated optical gyroscopes. In 2002, he was appointed to a lectureship in the Department of Electronics and Electrical Engineering, University of Glasgow. His research activities include interferometry, locking phenomena, semiconductor lasers, and integrated devices.

Alessandro Scirè was born in Rimini, Italy, on June 26, 1971. He received the S.M and Ph.D. degrees in electronic engineering and the S.M. degree in physics from the University of Pavia, Pavia, Italy, in 1995, 1999, and 2003, respectively.

He held a Marie Curie Individual Fellowship in 2000-2002 at the Instituto Mediterraneo de Estudios Avanzados (IMEDEA), Esporles, Spain, where he is presently working. His research interests include laser dynamics, stochastic processes in nonlinear systems, cooperative phenomena in coupled oscillators, and dynamic networks. 